\title{
Psychodrama mit Kindern und Jugendlichen
}

\author{
Christian Stadler $\cdot$ Kate Kirk
}

Online publiziert: 20. Januar 2017

(C) Springer Fachmedien Wiesbaden 2017

Das vorliegende Heft widmet sich den Kindern und Jugendlichen, also neben der Gegenwart unserer Zukunft. Wir versuchen dieses Mal größere - internationale Brücken zu schlagen. Dies zeigt sich schon in der Herausgeberschaft: Kate Kirk konnte von uns als Gastherausgeberin gewonnen werden, die bereits den ZPS-Sonderband zu Forschung und Wissenschaft 2 mit herausgab.

Das Thema Kinder und Jugendliche haben wir seit ein paar Jahren geplant. Zuletzt fanden wir die Diskussionen zwischen deutschen, österreichischen und schweizerischen Kinder- und Jugendtherapeuten so anregend, dass wir hofften, wir könnten die Diskussion in einem Themenheft abbilden. Was auf Kongressebene gerade den Veranstaltern ein drittes Mal 2016 mit dem Kinderkongress in Basel gelungen ist, ist auf der Ebene der AutorInnen leider nicht gelungen. Dies war auch ein Grund, warum wir das ausschließlich deutschsprachige Gebiet verlassen haben. Wir haben die Perspektive erweitert, was sich auch in der Herkunft der AutorInnen zeigt. Wir haben jetzt - trotz Brexit - drei Beiträge aus Großbritannien, - trotz Trump - zwei USamerikanische Beiträge, und - trotz Erdogan - einen türkischen Beitrag. Österreich, Schweiz und Deutschland sind selbstverständlich auch im Heft zu finden.

Wir haben mit den Artikellängen experimentiert, wir haben zwei Sprachen, wir haben Forschung und wir haben den Beginn eines Peer-Review-Verfahrens zur Qualitätssicherung der Beiträge. Peer-Review bedeutet, dass nicht (alleine) die HerausgeberInnen über die Aufnahme eines Beitrages entscheiden und den AutorInnen Feedbacks geben, sondern ein Pool externer ExpertInnen die Beiträge gesichtet und

C. Stadler $(\bowtie)$

Konrad-Adenauer-Str. 27, 85221 Dachau, Deutschland

E-Mail: praxisstadler@arcor.de

K. Kirk

CAMHS Noble's Hospital, Braddan Douglas, IM4 4DG, Isle of Man, UK

E-Mail: Kate.Kirk@gov.im 
nach Qualitätsstandards beurteilt hat. Das P-R-Verfahren wird in den kommenden Heften aus den Kinderschuhen befreit und in einen standardisierten Prozess überführt, damit wir auch für die akademisch tätigen WissenschaftlerInnen als ZPS an Attraktivität gewinnen.

\section{Von der Geburt bis zur Schule}

Neşe Karabekir schreibt über den Einsatz von Psychodrama bei der Geburt. Die Autorin beschreibt ihre Arbeit als Schwangerschafts- und Geburtspsychotherapeutin zusammen mit ihrem Team in Istanbul. Ihre Therapieform ist weltweit einzigartig. Gabriele Weiss setzt sich mit dem resilienzfördernden Einsatz psychodramatischer Methodik in Kindertageseinrichtungen auseinander. Im Rahmen psychodramatischer Angebote wird die seelische Widerstandsfähigkeit der Kinder gestärkt. Carl Dutton beleuchtet die Arbeit mit dem Psychodrama in Schulen. Rollen von PsychodramatikerInnen, die notwendig sind, um Psychodrama und Aktionsmethoden einzusetzen kommen zur Sprache ebenso wie die Lebendige Zeitung, Geschichten entwickeln und kreativ umsetzen und themenbasierte Vignetten. Auch Ruth Enz hat den Fokus Schule. Das von ihr beschriebene beziehungsfördernde Spiel erleichtert die Integration bzw. Inklusion möglichst aller SchülerInnen an öffentlichen Schulen. Mithilfe des Kinderpsychodramas werden Geschichten gespielt, in die SchülerInnen, LehrerInnen und anderes Fachpersonal einbezogen sind.

\section{Psychodrama in der Behandlung von Kindern und Jugendlichen}

Andrea Meents schildert in ihrer Fallstudie „Das Geheimnis des Pinguins“ die Teilearbeit mit Holztieren im Einzelsetting mit einem Kind mit Enkopresis. Rebecca Walters stellt Märchen, Bibliodrama, Soziodrama und Playback-Theater als Heilungswege für traumatisierte Kinder im stationären psychiatrischen Setting vor. Regine Reisinger beschreibt anhand ihrer Fallstudie mit einem 7-jährigen Mädchen aus einer konflikthaften Scheidungsfamilie, wie trotz der Schwere an der Resilienz gearbeitet werden kann. Juliane Markov zeigt, wie auf der Symbolebene soziales Kompetenztraining mit Psychodrama durchgeführt werden kann. „Im Computerspiel bin ich der Held“", sagt der depressive Jugendliche, mit dem Christoph Zauner im Einzelsetting mit selbstgestalteten Heldenfiguren ressourcenorientiert arbeitet.

\section{Kinder im Krankenhaus}

Kate Kirk zeigt exemplarisch an vier Fallstudien, wie mit Kindern komplexe, hilfreiche Geschichten erfunden werden können, die als tagklinische PatientInnen vom Psychodrama à deux profitieren. Leonhard Thun-Hohenstein und Julia TrostSchrems beschreiben ihre psychodramatische Arbeit an einer Kinder- und Jugendpsychiatrischen Versorgungs- und Universitätsklinik. Psychodrama ist dort fixer Bestandteil des Behandlungskonzeptes. Die Jugendlichen profitieren oft bereits 
durch sanfte und einfache Techniken wie Aufwärmübungen. Wie Ente, Affe, Frosch und Schweinchen mit der Angst umgehen erklärt Hannelore Houdek in ihrer Arbeit mit Kindern, die mithilfe von Intermediärobjekten auf eine Operation vorbereitet werden.

\section{Kinder- und Jugendlichen-Psychodrama aus der Metaperspektive}

Alfons Aichinger zeichnet die Entwicklung des Kinderpsychodramas in Deutschland im Format Gruppentherapie, Einzeltherapie, Familientherapie und Resilienzförderung in Kindergärten, Schulen und mit Risikogruppen nach. Das psychodramatische Spiel in der Kindertherapie hilft beim Mentalisieren, so Reinhard Krüger. Er unternimmt eine Einordnung des Kinderpsychodramas von Aichinger und Holl, Pruckner, Biegler-Vitek und Wicher. Dabei legt der Autor die Theorien von Fonagy und Schacht zugrunde. Karl-Ernst Heidegger widmet sich der Frage nach der Beziehungsgestaltung und Begegnung auf der Spielbühne. Er diskutiert dabei Probleme, wie die Rollenkonfiguration auf der Spielbühne aussieht sowie die Schwierigkeit, wenn Spiel- und PsychotherapeutInnenrolle gleichzeitig eingenommen werden. Christian Stadler fokussiert das Thema der Generationen, und wie dabei Eltern und Kinder in komplexe Lagen geraten können, und die herkömmliche Aufeinanderfolge der Generationen verstören. Roger Schaller schreibt schließlich darüber, wie das Kinder- und Jugendlichen-Psychodrama beforscht werden kann. Er gibt konkrete Anregungen für eine praxisorientierte Forschung mit dem Forschungsinstrument EQUALS.

Die Rubrik „Der andere Beitrag“ wird von den beiden US-Amerikanerinnen Erica Hollander und Amna Jaffer bedient. In ihrem Beitrag zur Förderung mit dem Psychodrama beschreiben sie ein Gruppentraining, das für 60 sehr unterschiedliche Teammitglieder einer Erziehungseinrichtung angeboten wurde. Diese MultiplikatorInnen wurden psychodramatisch geschult, damit sie unterprivilegierten Menschen eine bessere Schul- und Collegeausbildung ermöglichen.

Michael Schacht hat den Nachruf auf Zerka T Moreno geschrieben. Beim Lesen seiner Zeilen wird die wichtige Psychodramatikerin und letzte Ehefrau Morenos noch einmal für einen kurzen Moment lebendig.

Wir wünschen Ihnen mit dem internationalen Kinderheft einen anregenden Lesegenuss!

Christian Stadler und Kate Kirk 